\title{
L'École normale Saint-Joseph de Hull, 1909-1968 : 60 ans de formation d'institutrices
}

\section{Andrée Dufour}

\section{RÉSUMÉ}

Cet article est consacré à l'évolution d'une école normale de filles située dans l'Outaouais québécois et tenue de 1909 à 1968 par des religieuses, les Sours Grises de la Croix d'Ottawa, comme on les nommait alors. L'étude se penche sur le contexte éducatif de l'époque, sur la formation que recevaient les normaliennes, sur ses dirigeants et son personnel enseignant, sur ses programmes d'études et sur les brevets que l'institution offrait. Appuyée notamment sur les Annuaires de l'École normale, l'étude fait aussi largement état des effectifs étudiants, de leurs lieux et milieux d'origine, des diplômes postulés et de leur réussite. En définitive, on peut conclure que l'École normale Saint-Joseph de Hull offrit une formation qui répondait aux exigences du département de l'Instruction publique, aux attentes et aux besoins du milieu ainsi qu’aux aspirations des normaliennes. Cette expérience, qui aura duré six décennies, apparaît en définitive une réussite éducative.

\section{ABSTRACT}

This article explores the history of a girls' normal school-Saint-Joseph de Hull-located in Quebec's Outaouais region and run, between 1909 and 1968, by the religious order then known as les Sours Grises de la Croix d'Ottawa. It discusses the school's staff and teachers, the programs and diplomas offered, and the training received by its students, all within the educational context of the era. Drawing on the school's yearbooks, the author also takes inventory of the girls who studied at the school, examining their numbers, places of origin and social backgrounds, as well as their rate of success. In light of this analysis, the article argues that the École normale Saint-Joseph de Hull offered training that not only met the demands of the Ministry of Public Instruction, but also responded to the expectations of the field and the aspirations of the students themselves. This state of affairs, which would last nearly six decades, appears in hindsight to have been a definite educational success.

Institutions d'enseignement destinées à la formation des maîtres des écoles primaires et secondaires du Québec de 1857 à 1974, les écoles normales ont joué un rôle important dans l'histoire de l'éducation. Peu d'écrits leur ont cependant été consacrés. Après le livre d'Adélard Desrosiers qui publie en 1909 un ouvrage intitulé Les Écoles normales primaires de la province de Québec et leurs auvres, ${ }^{1}$ apparaît plusieurs 
décennies plus tard, en 1981, l'importante étude de Jeannette Létourneau, une synthèse sur Les écoles normales de filles au Québec. ${ }^{2}$ Plus récemment, dans un ouvrage publié en 1995, Un siècle de formation des maîtres au Québec, 1836-1939,3 Thérèse Hamel trace un portrait d'ensemble fort intéressant de ces maisons d'enseignement qui ont particulièrement marqué l'éducation au Québec durant plus d'un siècle. ${ }^{4}$

De très rares monographies ont porté sur l'étude d'une école normale en particulier. ${ }^{5}$ Mentionnons celle de Réal Bertrand concernant l'École normale Laval, de Québec, ${ }^{6}$ une école normale d'État qui n'admettait que les jeunes hommes. ${ }^{7}$ Mentionnons également celle de Georgette Grand'Maison sur l'école normale accueillant les jeunes filles à Sainte-Rose de Dégelis. ${ }^{8}$ Il s'agit, à notre connaissance, de la seule étude consacrée à une école normale de filles au Québec.

Ce texte se propose d'ajouter à la connaissance de ces nombreuses institutions d'enseignement - il y en eut 71 — en examinant l'établissement et le fonctionnement d'une des plus anciennes écoles normales destinées aux jeunes filles du Québec, l'École normale Saint-Joseph de Hull créée en 1908. Il s'agit de fait, de la septième école normale de filles établie au Québec, la toute première dans l'ouest de la province. Sa fondation s'inscrit dans la deuxième génération de ces écoles, durant la période qualifiée par Jeannette Létourneau, de " développement modéré " des années 1899 à 1939 durant laquelle 21 écoles normales destinées aux jeunes filles apparurent dans la province.

Auparavant, au XIX siècle, l'École normale des Ursulines de Québec, établie en 1857 lors de la mise sur pied des premières écoles normales du Canada-Est par le surintendant de l'éducation d'alors, Pierre-Joseph-Olivier Chauveau, fut longtemps la seule maison d'enseignement assurant la formation des futures enseignantes. Les autres jeunes filles désireuses d'enseigner devaient réussir l'examen d'un des Bureaux d'examinateurs de district mis sur pied par le surintendant Jean-Baptiste Meilleur en 1849. Au cours des années 1850, la grande majorité des comtés de la province étaient pourvus d'un bureau d'examinateurs. En 1898, les Bureaux d'examinateurs, dont on déplorait les insuffisances, notamment, le " caractère essentiellement théorique de la pédagogie " que devaient maîtriser les candidats et des exigences de certification très variables, seront remplacés par un organisme unique que l'on voulait plus cohérent, le Bureau central des examinateurs. ${ }^{9}$ Cette création avait pour but de relever les exigences d'admission dans la profession enseignante. ${ }^{10}$ Selon la première circulaire émise par le Bureau central des examinateurs catholiques, les aspirants aux brevets de capacité "n'étaient pas tenus de se rendre à Québec pour y être examinés ". De fait, les candidats et candidates à l'enseignement pouvaient se rendre dans quelque 30 localités, des localités où existaient auparavant des bureaux d'examinateurs de districts. ${ }^{11}$

La création du Bureau central n'avait cependant pas entraîné que des modifications mineures aux programmes de pédagogie et d'examen. Par ailleurs, les femmes étant nombreuses à enseigner dans les petites écoles, les évêques vont fortement appuyer la création d'écoles normales pour les filles par les communautés religieuses féminines. On assista donc à l'apparition rapide de bon nombre de ces écoles de filles d'abord situées à Montréal puis dans les métropoles régionales du Québec. C'est ainsi 
qu'après Rimouski, Chicoutimi, Trois-Rivières et Nicolet, fut créée à Hull l'École normale Saint-Joseph en $1908,{ }^{12}$ l'objet de ce texte.

Notre objectif est de vérifier si cette école offrit une formation qui répondait aux exigences du département de l'Instruction publique de même qu'aux attentes et aux besoins du milieu ainsi qu'aux aspirations des normaliennes. Pour ce faire, nous nous sommes attardée aux circonstances entourant la création de cette École, à son évolution de 1908 à 1968, à ses dirigeants et à son personnel enseignant ainsi qu'aux programmes d'études et aux brevets qu'elle offrait. Les étudiantes seront largement au cour de notre texte. Nous ferons état de leurs effectifs, de leurs lieux et milieux d'origine, de leur réussite et des diplômes qu'elles postulaient. Nous chercherons aussi à savoir si elles ont pu mettre à profit leur formation de normaliennes.

Pour ce faire, nous avons principalement consulté les Annuaires de l'École normale conservées aux Archives de l'École Saint-Joseph de Hull (AESSJH). Nous avons aussi fait appel à des programmes souvenir et à des documents publiés à l'occasion de conventum et d'amicales. Les informations fournies par deux anciennes directrices de l’École, Sœurs Clotilde Maurice et Rita Denis, ont aussi été précieuses.

\section{Création de l'École Saint-Joseph par les Sœurs Grises de la Croix d'Ottawa}

Les évêques furent des acteurs importants dans la mise sur pied de maintes écoles normales. En effet, ce sont eux qui, de concert avec les religieuses enseignantes, prirent souvent l'initiative de l'établissement d'écoles normales diocésaines de jeunes filles. ${ }^{13}$ Les sources consultées ne nous ont toutefois pas permis de mesurer l'influence de l'évêque du diocèse d'Ottawa, dont faisaient partie les paroisses de la ville de Hull depuis 1875, sur la fondation de l'École normale Saint-Joseph de Hull.

Quoiqu'il en soit, les Sœurs Grises de la Croix d'Ottawa ${ }^{14}$ communauté enseignante créée en 1866 et présentes dans cette ville depuis $1869,{ }^{15}$ furent largement à l'origine de la création de l'École normale. On lit en effet dans les archives de cette congrégation que l'institution fut fondée à la suite d'une entente survenue entre le Conseil de l'Instruction publique et la communauté religieuse, et ce, "à l'avantage plus particulier des comtés de Pontiac et d'Ottawa ". ${ }^{16}$

Sans doute les religieuses s'engagent-elles, comme les autres communautés, " à construire et à maintenir l'école, à « recevoir et à donner la pension aux jeunes filles désireuses d'enseigner ". Les écoles normales de filles étant moins subventionnées que celles des garçons, ${ }^{17}$ ces exigences s'avéraient très lourdes financièrement. La tenue d'une école normale constituait, selon Mgr J-A. Archambault, évêque de Joliette, " un acte de dévouement " à la cause de l'éducation primaire. ${ }^{18}$

Un octroi gouvernemental, prévu au contrat signé en juin 1908, aide cependant la communauté à édifier et à achever à l'été 1909 une importante construction de pierres voisine de leur ancien couvent Notre-Dame-de-Grâces comme le montre une photo illustrant les modifications apportées à l'édifice. À l'automne, l'École normale accueille ses premières élèves, au nombre de $38 .{ }^{19}$ Une photographie, prise en 1909 , nous montre le corps professoral et quelques étudiantes de cette première cohorte. 


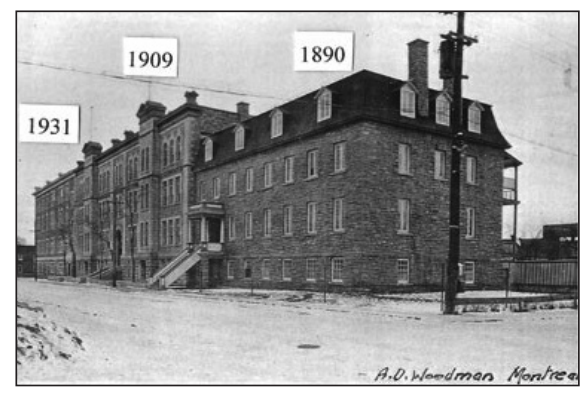

Photographie de 1931 de l'édifice abritant notamment, au centre, l'École normale de Hull (Archives des Socurs de la Charité d'Ottawa (ASCO), M18-Sa-SSI-D03.001).

\section{Évolution de l'École}

L'augmentation des élèves nécessite l'érection d'une nouvelle aile achevée en 1931, dotant entre autres les normaliennes d'une " magnifique salle académique ", où étaient jouées les pièces de théâtre et les saynètes, présentés des concerts, où avait lieu la remise des diplômes et, fait assez étonnant pour l'époque, d'un gymnase. ${ }^{20}$ En 1939, l'École innove également en accueillant des externes. Il s'agissait de "répondre aux besoins particuliers du milieu ", notamment de la croissance rapide de la population hulloise en raison de la croissance de la fonction publique à Ottawa ${ }^{21}$ et de permettre à d'éventuelles candidates " issues de familles peu fortunées " d'accéder à la profession d'institutrices. ${ }^{22}$ Réussie, l'expérience d'externats-écoles normales se

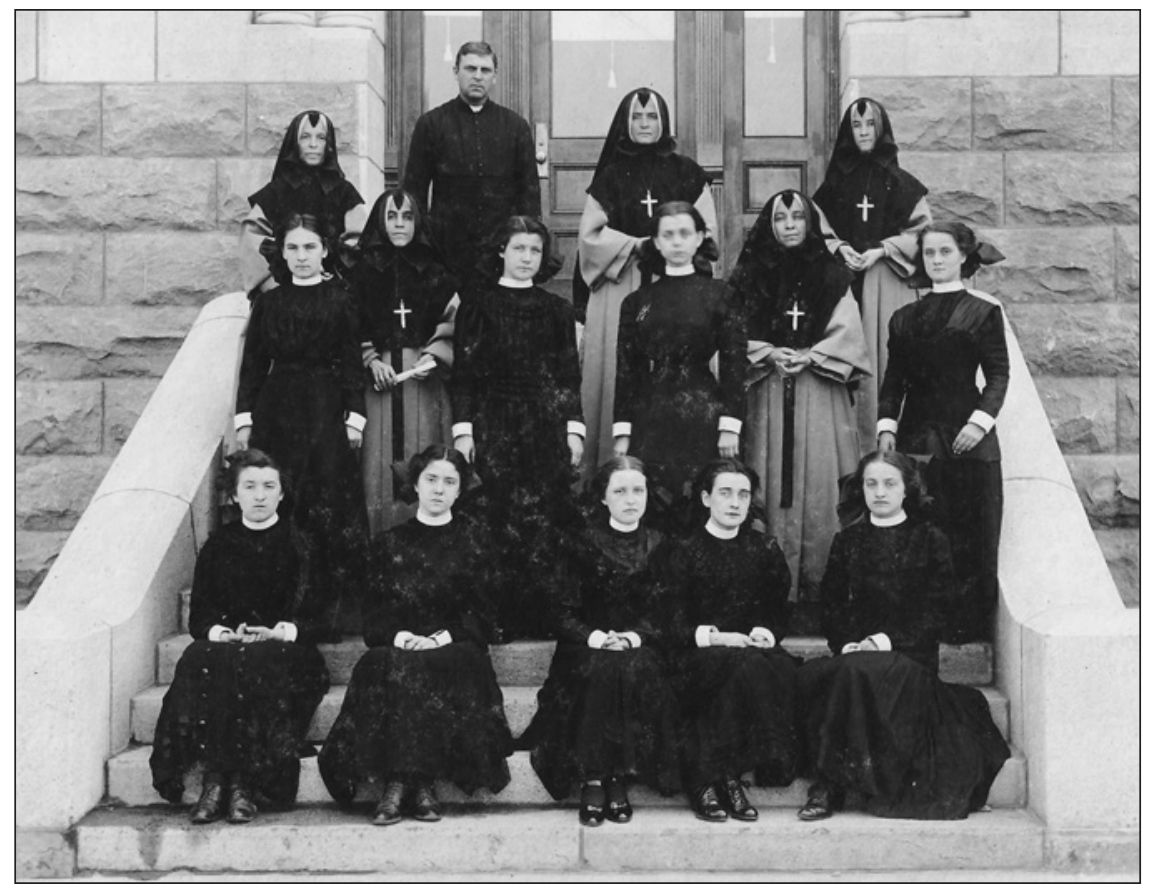

Corps professoral et étudiantes de l'École normale Saint-Joseph de Hull en 1909 (ACSO, P-M18-008). 
répètera d'ailleurs après 1950 dans les nouvelles écoles normales fondées dans les petites et les grandes villes du Québec. ${ }^{23}$

Malgré une situation financière plutôt difficile et un violent incendie survenu en 1950, l'École connaît une expansion notable de 1940 à 1960. Elle attire une clientèle nombreuse qu'elle doit même limiter dès $1945 .{ }^{24}$ Mais la recommandation de la Commission royale d'enquête sur l'enseignement dans la province de Québec, dite Commission Parent, recommande dans son Rapport publié en 1964 de confier la formation des maîtres aux universités. Cette recommandation amène des changements importants puis la fermeture définitive de l'École normale Saint-Joseph de Hull en juin 1968, après quelque 60 ans de fonctionnement ininterrompu.

\section{Programmes d'études et brevets d'enseignement}

L'École normale des Sœurs Grises de la Croix dépendait du département de l'Instruction publique (DIP) de la Province de Québec. Elle offrait donc les programmes d'études élaborés par ce dernier à l'intention des jeunes filles.

Ainsi, au début du $\mathrm{XX}^{\mathrm{e}}$ siècle, les normaliennes devaient pour être acceptées à l'École avoir complété huit années d'études, soit les quatre années du cours élémentaire, les deux années du cours modèle et les deux années du cours dit académique considéré comme de niveau secondaire. Elles se voyaient offrir trois différents brevets qui correspondaient au niveau d'enseignement postulé : élémentaire, modèle et académique. Le brevet élémentaire obtenu après un an d'études leur permettait d'enseigner au niveau élémentaire ; le brevet modèle obtenu après une deuxième année d'études normales les autorisait à enseigner aux enfants inscrits au cours modèle ; enfin le brevet académique qui couronnait une troisième année d'études normales leur permettait d'enseigner aux élèves inscrits au cours académique. ${ }^{25}$

Retrouvés dans les Annuaires de l'École, les «Tableaux de l'emploi du temps » de l'année scolaire 1909-1910 montrent l'horaire prescrit aux normaliennes inscrites aux trois différents brevets d'enseignement, soit élémentaire, modèle et académique. Celles-ci suivaient, selon un horaire bien précis, des cours nombreux et variés déjà précisés par le règlement de 1856 créant les écoles normales et redéfinis dans le nouveau programme d'études de $1905 .{ }^{26}$ À toutes les normaliennes de de l'année scolaire 1909-1910 étaient enseignés l'arithmétique, l'anglais, la comptabilité, le dessin, l'écriture, le français, l'histoire, la géographie, l'instruction civique, la lecture et la littérature, les sciences et la sténographie. Des cours de pédagogie étaient aussi donnés. Les élèves des cours modèle et académique recevaient de surcroît des cours de tenue des livres et de toisé. Enfin, aux élèves du cours académique étaient aussi enseignées la philosophie, la poésie et l'analyse littéraire comme le montrent les Tableaux de l'emploi $d u$ temps des cours élémentaire, modèle et académique. ${ }^{27}$

On trouve aussi dans les Archives de la communauté un règlement dactylographié annoté précisant minutieusement l'horaire journalier et hebdomadaire des normaliennes pensionnaires à Saint-Joseph, un horaire où apparaît le caractère religieux de leur formation. ${ }^{28}$ Ces tableaux et le règlement de la maison ne précisent pas le contenu de ces matières, mais on peut penser que le personnel enseignant de 
l'École s'inspirait des Règlements du Comité catholique du Conseil de l'Instruction publique, qui déterminait les matières à examens pour les trois brevets que pouvait décerner le Bureau central des examinateurs. ${ }^{29}$

Au total, les périodes de cours et d'études individuelles totalisaient plus de 40 heures, habituellement situées de $8 \mathrm{~h}$ à 16 h. Avant et après le souper, car les élèves étaient cette année-là encore obligatoirement pensionnaires, s'ajoutaient des périodes qui pouvaient être consacrées à la collation et aux promenades, mais aussi à la conversation anglaise, à la gymnastique et aux jeux, à la déclamation, au chant, à l'" accompagnement sur orgue » et, si nécessaire, à l'étude. Il s'agissait d'un horaire bien chargé où était toutefois absent l'enseignement du latin, pourtant ajouté au programme de formation des maitres en 1905. En contrepartie, la « coupe d'habits », bien que n'apparaissant pas aux tableaux, faisait partie du programme d'études. ${ }^{30} \mathrm{La}$ dactylographie et la sténographie étaient aussi enseignées. Était-ce une demande du milieu ? On peut le penser.

La pédagogie était présente dans la formation des normaliennes, de l'École SaintJoseph. Les Tableaux de l'emploi du temps de l'année 1909-1910 des cours élémentaire, modèle et académique montrent qu'aux cours théoriques des mercredis et vendredis était ajoutée une heure consacrée à la "Pratique de l'enseignement et [de l'] observation à l'École Modèle ». Il s'agissait d'une "École d'application " annexée à l'École normale où toutes les normaliennes pouvaient s'exercer à leur future profession. Une période de "critique ", probablement de commentaires sur l'attitude de l'élève institutrice, suivait cette pratique. Logée sous le même toit que l'École normale, l'École d'application dite aussi " annexe " comptait, en 1909, 78 élèves, réparties dans les quatre classes du cours élémentaire. ${ }^{31}$ Il nous apparaît donc difficile de souscrire entièrement à l'idée que la formation des normaliennes s'acquérait "principalement sur le tas » et qu'il «faudra attendre 1953 " pour que la formation professionnelle gagne véritablement une place spécifique dans les programmes. ${ }^{32}$

Les Annuaires de l'École ne reprennent malheureusement plus, après 1909, l'horaire des étudiantes. Ils nous ont cependant permis, avec les journaux locaux et les brochures publiées lors de grands événements, de suivre les modifications majeures apportées au cycle d'études de l'École. Ainsi, en septembre 1923, y était adoptée la nouvelle organisation pédagogique des écoles primaires et des écoles normales décidée par le département de l'Instruction publique. L'école publique n'offrait désormais que deux niveaux d'études : le cours élémentaire d'une durée de six ans, et le cours complémentaire de deux ans, de niveau secondaire selon les critères actuels. L'École normale s'adapta à ces nouvelles structures en n'offrant plus que deux possibles brevets. ${ }^{33}$ Ainsi, après avoir suivi ces huit ans d'études et obtenu son diplôme d'études primaires, la normalienne pouvait, après deux années passées à l'école normale dans les classes d'Élémentaire I et d'Élémentaire II, obtenir un brevet élémentaire. Une troisième année d'études, dite Cours supérieur, lui permettait d'accéder au brevet supérieur d'enseignement. ${ }^{34}$

Comme il s'agissait d'une école normale de jeunes filles, l'enseignement ménager était ajouté à la formation comme le montre le règlement de l'École. Une telle formation comprenait l'éducation familiale, l'étude théorique et pratique de la coupe, 
de la couture, de la cuisine, de la tenue de maison ainsi que des notions raisonnées d'hygiène et de médecine pratique. ${ }^{35}$

La durée de la formation offerte à l'école publique québécoise fut portée à 11 ans en 1929 puis à 12 ans en septembre 1937. ${ }^{36}$ Ainsi, en 1929, un nouveau niveau d'études dit "supérieur " d'une durée de trois ans fut ajouté aux études primaires d'une durée de six ans et aux études secondaires qui nécessitaient deux années supplémentaires. En 1936, la durée du cours élémentaire passa à sept années d'études auxquelles s'ajoutaient deux années pour l'obtention du diplôme complémentaire et trois années pour recevoir le diplôme dit supérieur. Des modifications survinrent également dans la formation offerte par les écoles normales par la mise en application, en 1938, d'un troisième programme provincial d'enseignement normal. Ce programme obligeait la future institutrice à se doter d'une « solide culture personnelle par l'étude approfondie de chacune des matières à enseigner ". À cette fin, comme le rappelait l'historienne Jeannette Létourneau, elle devait donc, sur le plan des connaissances, notamment s'appliquer :

" à rectifier au besoin, à compléter, à consolider ses connaissances et « à y mettre plus à y mettre plus de précision, de clarté et d'ordre ;

à donner des développements plus amples à ses connaissances déjà acquises en vue d'une plus complète intelligence de la matière, d'une meilleure assimilation et d'une grande facilité de transmission ;

à élever et à varier les différents aspects sous lesquels peuvent s'envisager les divers sujets que présente une matière ${ }^{37}{ }^{37}$

La formation en école normale devait également comprendre une formation professionnelle. Un programme de pédagogie nouveau fut défini. Il comprenait :

une étude approfondie des principes généraux de la pédagogie ;

une connaissance suffisante de la nature de l'enfant ;

l'étude de la psychologie appliquée à l'éducation ;

la connaissance théorique et une application pratique des méthodes, modes, procédés nécessaires à un enseignement gradué, méthodique, vivant et intéressant. ${ }^{38}$

Pour mieux répondre aux nouveaux programmes offerts à l'école publique et aux attentes du DIP, l'École normale Saint-Joseph modifia ses propres programmes d'études. La durée maximale de la formation pédagogique fut portée à quatre ans : aux cours élémentaires I et II qui, terminés, permettaient à la normalienne de se mériter le brevet élémentaire, s'ajoutaient les cours complémentaire et supérieur chacun d'une durée d'un an et couronnés d'un brevet du même nom. ${ }^{39}$ C'est ainsi qu'à la fin de l'année scolaire 1953-1954, l'École attribua le diplôme supérieur à neuf finissantes que l'on voit en toge blanche, coiffées du mortier et entourant le principal de l'École. ${ }^{40}$ 


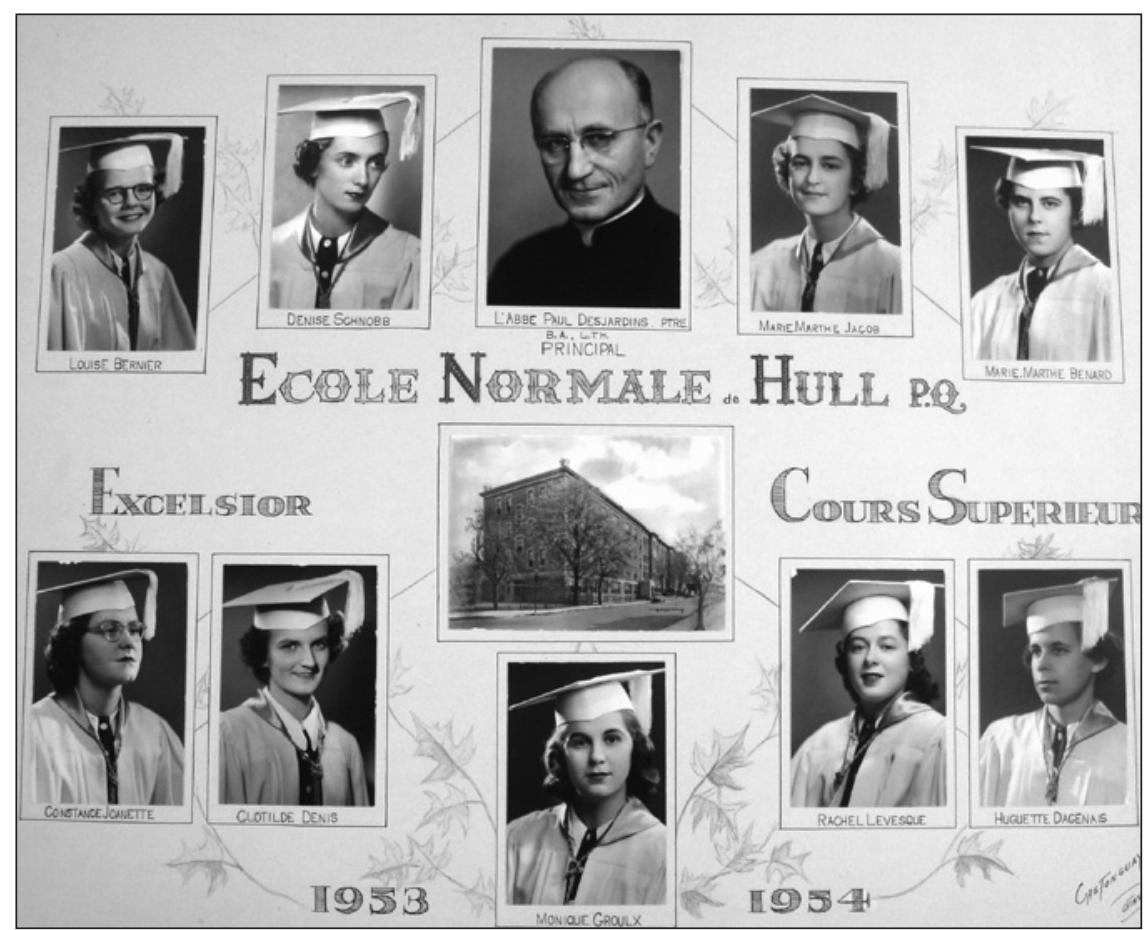

Finissantes de l'année scolaire 1953-1954 entourant le principal de l'École normale (P-M18-242, ASCO).

En 1954, le département de l'Instruction publique apporta à nouveau d'importants changements à la nomenclature et à l'organisation du cours normal. Le programme se voulait plus relevé. La formation en école normale devait même compléter les connaissances manquantes des candidates et des candidats, dont le sérieux était vérifié par un test de personnalité ${ }^{41}$ et, bien sûr, correspondre aux nouveaux programmes scolaires qui devaient être implantés au Québec en 1956. Ces programmes prévoyaient le maintien du cours élémentaire d'une durée de sept ans d'études dont la réussite était couronnée par un certificat d'études primaires, mais le cours complémentaire et le cours supérieur étaient regroupés et formaient désormais le cours secondaire d'une durée de cinq années au terme desquelles les élèves pouvaient obtenir un diplôme d'études secondaires. ${ }^{42}$ De nouveaux brevets d'enseignement furent établis, désignés par les lettres $\mathrm{C}, \mathrm{B}$ et $\mathrm{A}$. Le brevet $\mathrm{C}$ permettait d'enseigner de la $1^{\text {re }}$ à la $7^{\mathrm{e}}$ année, le brevet $\mathrm{B}$, de la $1^{\text {re }}$ à la $9^{\mathrm{e}}$ année et le brevet $\mathrm{A}$ de la $1^{\mathrm{re}}$ à la $12^{\mathrm{e}}$ année.

Ces grandes transformations furent rapidement appliquées à l'École Saint-Joseph. Ainsi, en même temps que les derniers brevets supérieurs, les premiers brevets " $\mathrm{C}$ " étaient accordés aux finissantes de juin 1956. ${ }^{43}$ À la fin de l'année scolaire 19601961, 100 nouvelles diplômées recevaient du département de l'Instruction publique, après un séjour d'un an, deux ans ou quatre ans à l'École normale, les brevets " $\mathrm{C}$ ", 
« $\mathrm{B}$ » ou « $\mathrm{A}$ ». ${ }^{44}$ Ce sont fort probablement des étudiantes du brevet A que l'on voit au laboratoire de chimie de l'institution durant l'année scolaire 1958-1959. Dès 1962 cependant, le brevet " $\mathrm{C}$ » était supprimé au Québec et n'était donc plus offert à l'École. En 1965, dans la foulée des grands travaux de la Commission d'enquête Parent ${ }^{45}$, le ministère de l'Éducation nouvellement créé ramena le cours primaire à six ans d'études et porta à cinq années l'obtention d'un diplôme d'études secondaires. L'École Saint-Joseph s'adapta à ce changement en continuant d'offrir aux détentrices d'un diplôme d'études secondaires, d'une $11^{\mathrm{e}}$ année désormais, une formation de deux ou de quatre ans menant aux brevets « $\mathrm{B}$ » et « $\mathrm{A}$ ».

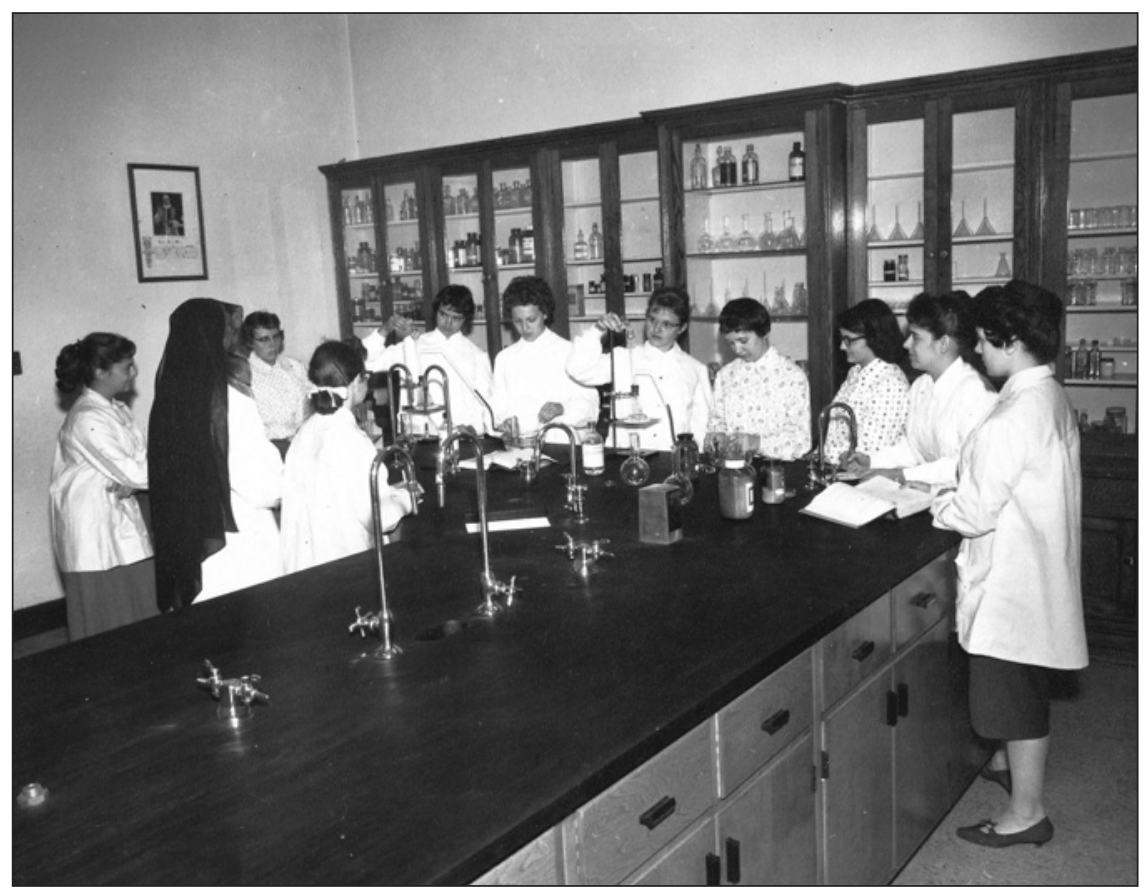

Laboratoire de chimie à l'École Saint-Joseph, année scolaire 1958-1959 (P-M18-071, ASCO).

Mais la fin des écoles normales approchait. Dont celle des nombreuses écoles accueillant les filles, objet de maintes critiques en dépit de l'uniformisation des programmes d'études à partir de 1953-1954. ${ }^{46}$ Dans un premier temps, les écoles normales furent fusionnées. Ainsi, à partir de septembre 1965, les normaliennes de Hull postulant le Brevet " $\mathrm{A}$ » durent suivre leurs cours à l'École normale de garçons de la ville, ouverte depuis 1961 seulement. ${ }^{47}$ L'École Saint-Joseph, conserva, elle, sous son toit les étudiantes inscrites au Brevet «B " jusqu’à la fin de ses activités, en juin $1968 .{ }^{48}$ Car désormais, les maîtres d'école devaient être tous et toutes formés à l'université, dans les facultés d'Éducation. 


\section{Le personnel enseignant}

Comme pour toutes les écoles normales de filles au Québec, le personnel enseignant de l'École Saint-Joseph présentait des caractéristiques particulières qui ont cependant évolué avec le temps. Ainsi, durant plusieurs décennies, les religieuses n'assumèrent pas le premier rôle dans la direction de leur École. À l'instar des écoles normales de filles du Québec, celle-ci était confié à un principal chargé, notamment, de l'admission des élèves et des cours de philosophie, de religion et d'histoire de l'Église. ${ }^{49}$ Un prêtre séculier occupât ce poste jusqu'en 1961 ; une religieuse de la communauté prit alors la relève, ${ }^{50}$ assurant enfin aux Sœurs Grises de la Croix la pleine responsabilité de l'organisation pédagogique de leur institution.

Comme l'exigeait aussi un règlement datant de $1899,{ }^{51}$ on retrouvait, au sein $\mathrm{du}$ personnel enseignant, un " professeur laïc ", nécessairement marié, respectable et d'âge mûr, auquel était toujours confié l'enseignement de la pédagogie et souvent des sciences et de l'histoire. ${ }^{52}$

Quant aux religieuses, bien que plus nombreuses que le personnel masculin, leur étaient surtout dévolus, dans les débuts les cours de couture, de dessin, de sténographie, par exemple ; de plus en plus qualifiées, elles devaient se charger à partir des années 1950 à tout le moins des matières dites "sérieuses " du programme d'études, telles que l'histoire, la géographie, la littérature, les mathématiques et même les sciences, comme le révèle l'Annuaire de l'École de 1953 et le suggère la photographie prise en 1958-1959, du laboratoire de chimie dirigée par une religieuse. ${ }^{53}$ Même si les sources disponibles ne permettent pas de le vérifier, les religieuses enseignantes de l'École normale ont probablement suivi l'exemple de leurs consœurs de Hull qui s'étaient dotées de diplômes de plus en plus élevés pour enseigner aux étudiantes de leur collège classique. ${ }^{54}$

La présence d'enseignantes laïques semble mince. Signalons tout de même qu'au début de la décennie 1950, une enseignante mariée fit son apparition à l'École, assurant le cours de diction. ${ }^{55}$

\section{Les normaliennes}

Les étudiantes de l'École Saint-Joseph ont connu maints changements, notamment sur le plan de leurs conditions d'admission, de leurs effectifs, de la poursuite de leurs études et de leur origine géographique et sociale. Il n'avait pas été facile de recruter les 38 premières élèves de l'École. Le principal du temps s'était même donné beaucoup de mal pour obtenir que des jeunes filles de Hull, des villages environnants ou apparentées aux religieuses de l'institution, munies d'un diplôme de $7^{\mathrm{e}}$ et de $8^{\mathrm{e}}$ année s'inscrivent à l'École normale. ${ }^{56}$ Il faut dire que les conditions d'admission étaient relativement nombreuses et pouvaient freiner la venue de certaines aspirantes. Les candidates devaient, en effet, remettre en premier lieu au principal un acte de baptême prouvant qu'elles avaient « seize ans révolus au mois de décembre qui suivra la rentrée des classes ", un certificat de moralité signé par le curé de même que le certificat d'un médecin " attestant qu'elle n'est pas atteinte d'aucune maladie de nature à 
la rendre impropre à l'enseignement ». Un examen devant le principal ou son délégué était en outre nécessaire si elles ne détenaient aucun diplôme du Bureau central des Examinateurs ou si elles désiraient s'inscrire à un cours dont le grade était " supérieur à celui qui lui a été décerné » par le dit Bureau. ${ }^{57}$ En 1929-1930, un âge minimum d'admission à l'école n'était plus requis, ${ }^{58}$ mais pour être admise en élémentaire l'élève devait, en plus de produire ses certificats, "subir avec succès devant le principal ou son délégué " un examen écrit "sur les matières de la $\sigma^{\mathrm{e}}$ année des écoles primaires élémentaires, portant notamment sur la langue française (grammaire, dictée, analyse, rédaction) et sur les mathématiques $" .^{59}$

L'enseignement n'était pas gratuit. Les frais de pension et de scolarité, dont le montant était fixé par le Comité catholique du Conseil de l'Instruction publique, ${ }^{60}$ s'élevaient à 75 \$ par année en 1909. Certaines jeunes filles recevaient toutefois une bourse d'études de $15 \$ .{ }^{61}$ Cette somme ne comprenait pas le coût des manuels scolaires, à la charge des parents. Vingt ans plus tard, la pension était portée à $120 \$$, mais la bourse demeurait la même. ${ }^{62}$ Par la suite, on peut supposer que la pension était passée, comme dans les autres écoles normales, à 150 \$ en 1943 et à $250 \$$ en $1949 .{ }^{63}$

Le port d'un uniforme était obligatoire. Une longue robe noire, unie, à col et poignets blancs était de mise pour les premières normaliennes, comme le montre

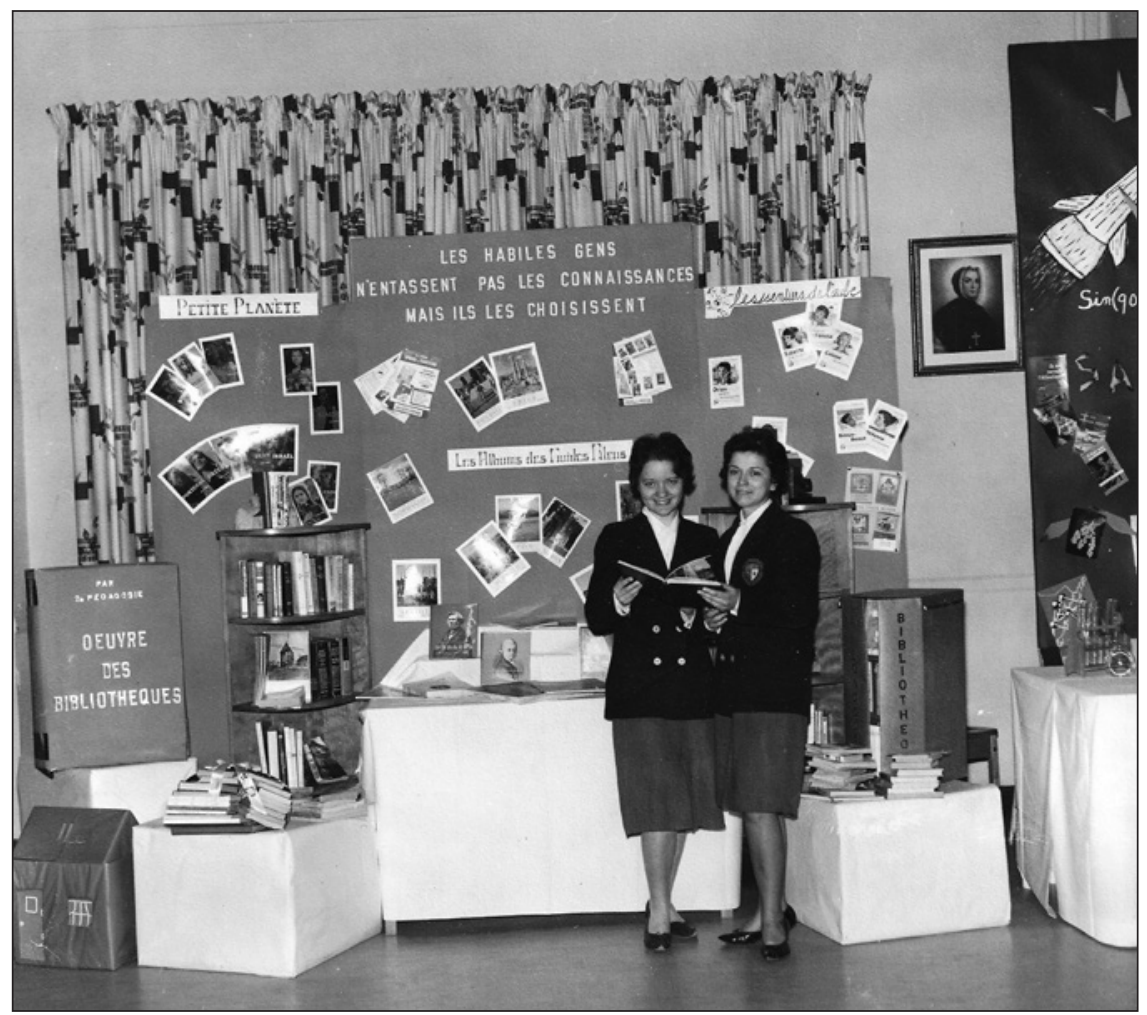

Exposition culturelle — Le Parchemin, activité parascolaire faite à bibliothèque en 1959-1960 (P-M18-312, ASCO). 
la photo de normaliennes et de religieuses enseignantes de $1909 .{ }^{64}$ Raccourcie peu à peu, elle devenait bleu marine au tournant des années 1940 et cédait finalement la place durant la décennie 1950 à la juge grise, à la blouse blanche et au blazer bleu, ${ }^{65}$ comme on peut le voir sur la photographie 5 de quelques étudiantes lors d'une activité parascolaire, une exposition culturelle faite à bibliothèque en 1959-1960. À noter que l'uniforme des normaliennes rappelle l'uniforme des étudiantes des collèges classiques. Comme ces dernières, les normaliennes de Saint-Joseph possédaient un journal, Le Parchemin.

\section{Évolution des effectifs scolaires}

Comme le montre le Tableau 1.1, qui reprend le nombre de normaliennes inscrites par année scolaire, ce nombre est peu élevé au départ, mais il connaît, une progression généralement continue de 1909 à 1933. Curieusement l'agrandissement de l'École en 1931 s'accompagne d'une diminution de l'effectif scolaire. On pourrait expliquer ce recul par la fondation par la communauté la même année de l'École normale Ville-Marie, située au Témiscamingue, où de 20 à 25 de leurs diplômées du cours supérieur désireuses d'enseigner passaient auparavant les examens du Bureau central des examinateurs.

Tableau 1.1

Nombre d'élèves inscrites et diplômées, par année scolaire et par niveau d'études, de 1909 à 1923

\begin{tabular}{|l|c|c|c|c|c|c|}
\hline \multirow{2}{*}{ Année scolaire } & \multicolumn{2}{|c|}{ Cours élémentaire } & \multicolumn{2}{c|}{ Cours modèle } & \multicolumn{2}{c|}{ Cours académique } \\
\cline { 2 - 7 } & Inscrites & Diplômées & Inscrites & Diplômées & Inscrites & Diplômées \\
\hline $1909-1910$ & 12 & 10 & 10 & 8 & 9 & 5 \\
\hline $1911-1912$ & 15 & 7 & 19 & 15 & 10 & 9 \\
\hline $1912-1913$ & 16 & 9 & 16 & 12 & 8 & 7 \\
\hline $1913-1914$ & 20 & 10 & 13 & 11 & 6 & 6 \\
\hline $1914-1915$ & 19 & 8 & 15 & 11 & 6 & 6 \\
\hline $1915-1916$ & 24 & 9 & 13 & 10 & 5 & 5 \\
\hline $1916-1917$ & 25 & 13 & 13 & 12 & 8 & 8 \\
\hline $1917-1918$ & 25 & 16 & 19 & 11 & 4 & 4 \\
\hline $1918-1919$ & 22 & 6 & 14 & 13 & 6 & 5 \\
\hline $1920-1921$ & 26 & 11 & 23 & 21 & 8 & 8 \\
\hline $1921-1922$ & 30 & 10 & 21 & 11 & 10 & 10 \\
\hline $1922-1923$ & 26 & 13 & 27 & 21 & 8 & 8 \\
\hline Total (en \%) & 260 & 109 & 203 & 156 & 88 & 81 \\
\hline
\end{tabular}

Source : ASSJH, Annuaires de l'École normale de Hull, 1909-1923. 
Nous n'avons pas de données pour les années 1933 à 1947. D’après le tableau 1.2, on constate cependant que 143 élèves sont inscrites à l'École normale en 1947-1948, soit plus que le double de l'année 1929-1930. La décision du gouvernement d'abolir en 1939 le Bureau central des Examinateurs catholiques et de confier aux seules écoles normales la formation des institutrices, jointe à l'acceptation, la même année, d'élèves externes amènent une accélération notable des effectifs, les normaliennes externes formant en moyenne $45 \%$ des élèves de 1949 à $1953 .{ }^{66}$ Par la suite, les inscriptions se maintiennent malgré la création, en 1945, par les Sœurs Grises d'un collège classique à Hull, le Collège Marguerite-d'Youville, et l'établissement, entre 1940 et 1946, de trois nouvelles écoles normales dans l'Ouest du Québec, soit à Amos, Chapeau et Saint-André-Avelin. Il faut dire que les besoins en institutrices sont grands depuis l'adoption de la loi de l'instruction obligatoire en 1940 qui oblige tous les enfants de 6 à 14 ans à fréquenter l'école. Aussi, la communauté qui hébergeait le Collège classique à l'École normale décide-t-elle en 1949 de récupérer ses locaux pour accueillir un nombre grandissant de candidates à l'enseignement. Quelques collégiennes ont pu poursuivre leurs études à l'École normale, le Collège Marguerite-d'Youville devant fermer provisoirement ses portes faute de locaux, car le nombre d'élèves terminant les cours Élémentaire II et Complémentaire augmente sensiblement.

Tableau 1.2

Nombre d'élèves inscrites et diplômées par année scolaire et par niveau d'études de 1923 à 1933

\begin{tabular}{|l|c|c|c|c|}
\hline \multirow{2}{*}{ Année scolaire } & \multicolumn{2}{|c|}{ Cours complémentaire II } & \multicolumn{2}{c|}{ Cours supérieur } \\
\cline { 2 - 5 } & Inscrites & Diplômées & Inscrites & Diplômées \\
\hline $1923-1924$ & 24 & 16 & 8 & 8 \\
\hline $1924-1925$ & 22 & 11 & 11 & 10 \\
\hline $1925-1926$ & 26 & 19 & 12 & 12 \\
\hline $1926-1927$ & 22 & 20 & 10 & 9 \\
\hline $1927-1928$ & 29 & 21 & 11 & 11 \\
\hline $1928-1929$ & 20 & 11 & 14 & 14 \\
\hline $1929-1930$ & 26 & 17 & 14 & 14 \\
\hline $1930-1931$ & 20 & 13 & 16 & 16 \\
\hline $1931-1932$ & 24 & 20 & 16 & 16 \\
\hline $1932-1933$ & 25 & 16 & 22 & 20 \\
\hline Diplômées (\%) & 238 & $164(68,9 \%)$ & 134 & 130 (97\%) \\
\hline
\end{tabular}

Source : AESSJH, Annuaires de l'École normale de Hull, 1923-1933.

À la distribution des prix de l'année scolaire 1951-1952, 166 élèves sont présentes. En 1952, toutefois, année de reprise des activités du Collège Marguerite-d'Youville, les inscriptions diminuent sensiblement. Une certaine concurrence entre les deux 
institutions pouvait exister, venant peut-être du désir de quelques jeunes filles d'accéder à l'université.

\section{Poursuite des études et réussite scolaire}

La majorité des élèves inscrites à la rentrée complètent leur année scolaire. Il arrivait même, comme en 1949-1950 et 1952-1953, qu'une ou plusieurs jeunes filles s'ajoutent au groupe en cours de route. Mais, de 1909 à 1923, lorsqu'existaient les brevets élémentaire, modèle et académique, on peut constater par le Tableau 1.1 qu'à l'exception de l'année d'ouverture, la majorité des élèves préféraient s'arrêter après l'obtention du brevet élémentaire ou modèle.

Le Tableau 1.2 illustre la même tendance au cours des années 1923 à 1933. Cependant quelques et parfois plusieurs normaliennes quittaient avant même d'obtenir le brevet élémentaire décerné seulement après la réussite des examens d'élémentaire II. C'est ainsi qu'on peut lire qu'en 1934, l'École normale avait vu " passer dans ses murs 800 élèves et accordé 710 brevets à 555 normaliennes $»{ }^{67}$ Des normaliennes échouaient les examens passés à l'École.

Par la suite, de 1947 à 1953 du moins, les normaliennes semblent, comme le montre le Tableau 1.3, tenir davantage à décrocher au moins le diplôme élémentaire II et même le complémentaire. L'exigence établie depuis 1911 d'avoir 17 ans révolus pour enseigner incite apparemment ici aussi plusieurs élèves à obtenir au moins un brevet. $^{68}$ Toutefois — et c'était d'ailleurs un phénomène général au Québec — une faible proportion des filles envisagent le diplôme supérieur. ${ }^{69}$ L'égalité des salaires quel que soit le brevet obtenu, ${ }^{70}$ la situation économique de la famille et, pour certaines normaliennes, la difficulté des examens ${ }^{71}$ et, on peut le penser, la courte durée de la carrière d'enseignante, expliquent en bonne partie l'arrêt précoce des études.

Tableau 1.3

Nombre d'élèves présentes à la distribution des prix, par année scolaire et par cours, de 1947 à 1953

\begin{tabular}{|l|c|c|c|c|c|c|}
\hline $\begin{array}{l}\text { Année } \\
\text { scolaire }\end{array}$ & $\begin{array}{c}\text { Élémentaire } \\
\text { I }\end{array}$ & $\begin{array}{c}\text { Élémentaire } \\
\text { II }\end{array}$ & $\begin{array}{c}\text { Complé- } \\
\text { mentaire }\end{array}$ & Supérieur & Total & $\begin{array}{c}\text { Inscrites en } \\
\text { septembre }\end{array}$ \\
\hline $1947-1948$ & 53 & 42 & 35 & 13 & 143 & \\
\hline $1948-1949$ & 44 & 53 & 31 & 13 & 141 & 144 \\
\hline $1949-1950$ & 48 & 49 & 27 & 13 & 137 & 136 \\
\hline $1950-1951$ & 47 & 61 & 22 & 10 & 140 & \\
\hline $1951-1952$ & 49 & 67 & 36 & 10 & 162 & 164 \\
\hline $1952-1953$ & 46 & 55 & 30 & 8 & 139 & 134 \\
\hline Total (en \%) & 291 & 326 & 181 & 75 & 865 & \\
\hline
\end{tabular}

Source : AESSJH, Annuaires de l'École normale de Hull de 1953. 
En juin 1962, la situation est essentiellement la même : 43 finissantes (81 \%) reçoivent un brevet « B ", et 10 (19\%) un brevet " A ». L'année suivante cependant, on note une évolution : 100 candidates se présentent au " $\mathrm{B}$ » et 48 au Brevet " $\mathrm{A}$ ". Près du tiers des candidates au brevet ont préféré doubler leur temps d'études et se doter du privilège d'enseigner de la $1^{\text {re }}$ à la $12^{\mathrm{e}}$ année. ${ }^{72}$ L'amélioration des salaires depuis 1960 motive aussi, sans doute les jeunes filles à rester à l'école normale. Il y a plus, selon un article intitulé " La Pédagogie ... à l'école normale St-Joseph de Hull » et publié en juin 1961 :

La profession d'institutrice est loin d'être encombrée. Les salaires sont intéressants, le travail nécessite du dévouement, du don de soi. Ces deux caractéristiques attirent beaucoup de finissantes du cours secondaire, qui chaque année s'inscrivent nombreuses à l'École normale. ${ }^{73}$

\section{Lieu d'origine des élèves}

Dans les Annuaires de l'École est noté le lieu d'origine des élèves inscrites de septembre 1909 à juin 1931 puis de septembre 1947 à juin 1952. Nous avons divisé cette provenance géographique en quatre groupes, soit : de la ville de $\mathrm{Hull}$, de l'Outaouais québécois comprenant surtout les nombreuses petites localités des comtés environnants, des régions éloignées du Québec, c'est-à-dire situées hors de la zone d'attraction de Hull, et pour finir de l'Ontario limitrophe. De 1909 à 1922, années durant lesquelles aucune autre école normale n'est établie dans l'Ouest de la province, $45 \%$ des candidates viennent de Hull, $40 \%$ de l'Outaouais, $11 \%$ des régions plus éloignées et $4 \%$ de l'Ontario. Ces proportions se modifient à partir de 1923 et jusqu'en 1930 du moins; avec le changement des structures pédagogiques puis l'établissement d'une école normale à Mont-Laurier et à Ville-Marie, $60 \%$ des élèves viennent de la ville et seulement $36 \%$ de l'Outaouais ou de régions plus lointaines. Le pourcentage d'élèves ontariennes n'est plus que de 3,8 \%.

Mais de 1947 à 1953, en dépit de l'admission d'externes et de la multiplication, dans le même temps, des écoles normales, notamment celle de Saint-André-Avelin créée en 1946, on note une sensible diminution des inscriptions hulloises (39\%) et une forte augmentation de la clientèle outaouaise et extérieure à la région $(61 \%)$. L'encombrement de la profession dans la ville et la bonne réputation de l'institution dans la région et au-delà pourraient expliquer ce nouveau renversement. Il faut dire aussi que des 162 élèves venant de régions éloignées, 100 (62\%) étaient originaires de Shawinigan, ville ouvrière située à plus de $350 \mathrm{~km}$ de Hull. Cette ville était dépourvue d'école normale, mais elle était pourtant située à seulement $40 \mathrm{~km}$ de TroisRivières où les Ursulines avaient ouvert une école normale depuis 1908. Cette École avait peut-être fait le plein d'inscriptions. Il y a aussi le fait que les Sours Grises de la Croix tenaient à Shawinigan un couvent et deux académies affiliées à l'Université Laval depuis 1945. 


\section{Persévérance scolaire et lieu d'origine}

D’où venaient les candidates aux brevets les plus élevés ? Le Tableau 2, qui reprend le pourcentage d'élèves inscrites en dernière année, au niveau académique puis supérieur selon le lieu de résidence familiale, montre que plus de Hulloises, de citadines, optaient pour les études les plus longues, particulièrement durant les années 1923 à 1931. Pour la période 1947 à 1953, bon nombre d'élèves originaires de Hull poursuivent leurs études au niveau supérieur, mais beaucoup moins de jeunes villageoises de la région et de pensionnaires venant de régions éloignées, en majorité originaires de la ville de Shawinigan, se retrouvent en fin d'études. Des raisons économiques et sociales, d'âge ou d'intérêt des élèves de ces milieux pour les études plus avancées peuvent expliquer un tel écart. Mais, le milieu urbain, peut-être plus exigeant à l'égard de ses institutrices et plus ouvert en ce qui concerne l'éducation des filles, pourrait avoir joué lui aussi un rôle dans la continuation des études. En contrepartie, le besoin d'institutrices suffisamment qualifiées pour enseigner aux tout jeunes enfants a pu être déterminant en milieu rural. En effet, les religieuses encourageaient plus volontiers les élèves des villes à approfondir leurs connaissances, tandis, qu'à l'opposé, le principal de l'École incitait les jeunes rurales à retourner chez elles sitôt le premier diplôme obtenu. ${ }^{74}$

\section{Tableau 2}

\section{Lieu d'origine des élèves inscrites au diplôme le plus élevé, par période}

\begin{tabular}{|l|c|c|c|c|c|}
\hline $\begin{array}{l}\text { Période } \\
\text { (brevet le plus } \\
\text { élevé postulé) }\end{array}$ & $\begin{array}{c}\text { Nombre } \\
\text { total des } \\
\text { élèves }\end{array}$ & $\begin{array}{c}\text { Élèves } \\
\text { de Hull }\end{array}$ & $\begin{array}{c}\text { Élèves de } \\
\text { I'Outaouais } \\
\text { québécois }\end{array}$ & $\begin{array}{c}\text { Élèves de } \\
\text { régions } \\
\text { éloignées }\end{array}$ & $\begin{array}{c}\text { Élèves de } \\
\text { I'Ontario }\end{array}$ \\
\hline $\begin{array}{l}\text { de sept. 1909 } \\
\text { à juin 1923 } \\
\text { (académique) }\end{array}$ & 88 & 44 & 31 & 10 & 3 \\
\hline $\begin{array}{l}\text { de sept.1923 à } \\
\text { juin 1931 } \\
\text { (supérieur) }\end{array}$ & 96 & 63 & 26 & 3 & 4 \\
\hline $\begin{array}{l}\text { de sept. 1947 à } \\
\text { juin 1953 } \\
\text { (supérieur) }\end{array}$ & 67 & 29 & 19 & 19 & 0 \\
\hline
\end{tabular}

Source : AESSJH, Annuaires de l'École normale de Hull de 1909-1921, 1922-1933 et 1953.

\section{Taux d'obtention des brevets des normaliennes.}

Dans un premier temps, nous avons pu relever le nombre de jeunes filles qui, de 1909 à 1923 puis de 1923 à 1933, réussissaient les examens du Bureau central préparés et passés à l'École et qui obtenaient ainsi leur brevet du département de l'Instruction publique.

En ce qui concerne l'obtention du brevet élémentaire, le taux de réussite était relativement peu élevé comme le montre le Tableau 1.1 : des 260 jeunes normaliennes inscrites de 1909 à 1923, que 122, soit 47 \% obtenaient leur diplôme. La jeunesse 
des candidates et le manque de préparation aux études normales pourraient expliquer un si faible pourcentage. Plus matures ou mieux préparées, les candidates des niveaux plus avancés réussissaient beaucoup mieux. Ainsi le taux de réussite des 203 normaliennes inscrites au cours modèle atteint $77 \%$, alors que ce taux est de $92 \%$ pour les 88 inscrites au cours supérieur. Comme il apparaît au Tableau 1.2, cette tendance se maintient de 1923 à 1933 chez les jeunes filles postulant pour l'obtention du diplôme complémentaire II et le diplôme du cours supérieur. En effet, 164 des 248 inscrites, soit $66 \%$ des postulantes, décrochaient un diplôme supérieur alors que 130 des 134 élèves $(97 \%)$ inscrites obtenaient leur diplôme supérieur.

À défaut de pouvoir comparer les pourcentages d'obtention des brevets des normaliennes de Saint-Joseph à ceux d'autres écoles normales, nous les avons mis en parallèle avec ceux des aspirantes et aspirants des mêmes brevets qui se présentaient au Bureau central des examinateurs catholiques. ${ }^{75}$ Même si elles ne concernent que les années 1909 à 1916 et 1923 à 1928, les données du Tableau 3 nous révèlent tout de même que les élèves formées à l'École normale Saint-Joseph réussissaient presque toujours mieux aux examens du Bureau central que l'ensemble des non normaliens et normaliennes du Québec, suggérant ainsi que une meilleure préparation des normaliennes aux examens.

\section{Tableau 3}

Pourcentage de réussite au brevet académique de 1909 à 1916 puis de 1924 à 1928 des normaliennes de l'École Saint-Joseph et des candidats au Bureau central des examinateurs

\begin{tabular}{|l|c|c|c|c|c|}
\hline $\begin{array}{l}\text { Année } \\
\text { scolaire }\end{array}$ & $\begin{array}{c}\% \text { de } \\
\text { réussite } \\
\text { à I'École }\end{array}$ & $\begin{array}{c}\text { \% de } \\
\text { réussite au } \\
\text { Bureau } \\
\text { central }\end{array}$ & $\begin{array}{c}\text { Année } \\
\text { scolaire }\end{array}$ & $\begin{array}{c}\% \text { de } \\
\text { réussite } \\
\text { à I'École }\end{array}$ & $\begin{array}{c}\text { \% de } \\
\text { réussite au } \\
\text { Bureau } \\
\text { central }\end{array}$ \\
\hline $1909-1910$ & $55,6 \%$ & $61,2 \%$ & $1916-1917$ & $100 \%$ & $83,5 \%$ \\
\hline $1911-1912$ & $90,0 \%$ & $85,5 \%$ & $1917-1918$ & $100 \%$ & $76,4 \%$ \\
\hline $1912-1913$ & $87,5 \%$ & $52,0 \%$ & $1918-1919$ & $83,0 \%$ & $86,1 \%$ \\
\hline $1913-1914$ & $100 \%$ & $77,6 \%$ & $1920-1921$ & $100 \%$ & $78,1 \%$ \\
\hline $1914-1915$ & $100 \%$ & $74,8 \%$ & $1921-1922$ & $100 \%$ & $89,5 \%$ \\
\hline $1915-1916$ & $100 \%$ & $79,7 \%$ & $1922-1923$ & $100 \%$ & $88,0 \%$ \\
\hline & & & & & \\
\hline $1923-1924$ & $100 \%$ & $68,2 \%$ & $1928-1929$ & $100 \%$ & $87,6 \%$ \\
\hline $1924-1925$ & $90,1 \%$ & $77,9 \%$ & $1929-1930$ & $100 \%$ & $82,9 \%$ \\
\hline $1925-1926$ & $100 \%$ & $83,4 \%$ & $1930-1931$ & $100 \%$ & $89,0 \%$ \\
\hline $1926-1927$ & $90,0 \%$ & $88,4 \%$ & $1931-1932$ & $100 \%$ & $82,7 \%$ \\
\hline $1927-1928$ & $100 \%$ & $82,7 \%$ & $1932-1933$ & $90,9 \%$ & $82,1 \%$ \\
\hline
\end{tabular}

Sources : AESSJH, Annuaires de l'École normale St-Joseph de Hull, 1923-1933, Jeannette Létourneau, Les Écoles normales de filles au Québec (Montréal : Fides, 1981), 214. 


\section{Origine sociale des élèves}

À quel milieu social appartenaient les jeunes filles fréquentant l'École Saint-Joseph ? Nous avons pu connaître l'origine sociale des 141 normaliennes inscrites en 19481949 aux niveaux élémentaire, complémentaire et supérieur et pour lesquelles est indiquée la profession du père. Les pourcentages obtenus ont été comparés à la répartition présente dans le Mémoire des collèges classiques féminins de 1954 pour l'ensemble des 15 collèges classiques féminins dont les catégories sociales étaient comparables. ${ }^{76}$ Le Tableau 4 fait état de cette comparaison. Nous avons cependant dû introduire un groupe supplémentaire relativement important à l'École normale, celui dont le père est décédé ou malade.

\section{Tableau 4}

\section{Origine sociale des normaliennes de l'École Saint-Joseph de Hull et des étudiantes de collèges classiques du Québec}

\begin{tabular}{|c|c|c|c|}
\hline $\begin{array}{l}\text { Groupes } \\
\text { Professionnels }\end{array}$ & $\begin{array}{l}\text { Normaliennes } \\
\text { de } 1948-1949 \\
\text { nombre } \%\end{array}$ & & $\begin{array}{c}\text { Étudiantes des collèges } \\
\text { du Québec en } 1954 \%\end{array}$ \\
\hline $\begin{array}{l}\text { Propriétaires, administrateurs } \\
\text { et professionnels }\end{array}$ & 25 & $17,8 \%$ & $79,8 \%$ \\
\hline $\begin{array}{l}\text { Employés de bureau et } \\
\text { de commerce }\end{array}$ & 13 & $9.2 \%$ & $10,4 \%$ \\
\hline $\begin{array}{l}\text { Gens de métier et } \\
\text { Ouvriers spécialisés }\end{array}$ & 29 & $20,6 \%$ & $7,6 \%$ \\
\hline $\begin{array}{l}\text { Ouvriers } \\
\text { non spécialisés }\end{array}$ & 26 & $18,5 \%$ & $0,2 \%$ \\
\hline Cultivateurs & 32 & $22,6 \%$ & $2,0 \%$ \\
\hline «Décédés, malades» & 16 & $11,3 \%$ & Non indiqué \\
\hline
\end{tabular}

Sources : AMPNDH, Liste des élèves de 1948-1949; Mémoire des collèges classiques féminins (Montréal : Fides, 1954)

Au premier coup d'œil, on peut observer une répartition assez équilibrée de l'origine sociale des normaliennes. Bien qu'elle ait été faite à quelques années d'écart et qu'elle ne concerne qu'une seule cohorte d'élèves, cette répartition montre un contraste frappant entre le milieu familial des normaliennes et celui des collégiennes du Québec Alors que près de $80 \%$ des jeunes filles fréquentant les collèges classiques venaient, nous l'avons déjà mentionné, de milieux dits "bourgeois " et devaient payer des droits de scolarité plus élevés, les normaliennes appartenaient principalement à des familles de conditions plus modestes et même défavorisées. Plus du quart ont un père cultivateur alors que la clientèle des collèges classiques vient surtout de milieux urbains. Peu d'entre elles ont un père "col blanc " et le père de plus de $11 \%$ des élèves est soit "décédé " $(15 \mathrm{cas})$, soit " malade " $(1 \mathrm{cas})$. On peut d'ailleurs penser 
que les religieuses venaient financièrement en aide à ces élèves et leur apportaient une aide soutenue, car sur 13 élèves inscrites au brevet supérieur, 5 dont 4 de Hull étaient orphelines de pères.

\section{Après l'école normale}

À l'automne de 1929, l'Amicale des Anciennes de l'École se réunit à l'occasion de son deuxième conventum. Il y était rappelé, entre autres, que depuis 1909, depuis 20 ans, 375 (75\%) diplômées de l'École avaient enseigné " dans les écoles rurales et urbaines ». Cela suggère que le quart des diplômées n'avaient pas enseigné, s'étant peut-être mariées ou avaient choisi une tout autre occupation. On rappelait aussi que 163 des 375 institutrices issues de l'École, quasi la moitié, mettaient encore à profit leur formation. ${ }^{77}$ Peut-être étaient-elles demeurées célibataires ou œuvraient à l'intérieur de communautés religieuses. La célébration du $25^{\mathrm{e}}$ anniversaire de la maison en 1934 suggère cette seconde hypothèse. On soulignait en effet, que parmi les 555 diplômées de l'École depuis sa fondation, 95 avait œuvré dans l'enseignement comme Sœurs Grises de la Croix et 19 avaient enseigné comme religieuses au sein d'autres communautés. ${ }^{78}$ Au total, 114 institutrices, soit 20,5 \% des diplômées, poursuivaient un idéal à la fois religieux et éducatif.

Quant à l'École normale elle-même, elle devint dès 1970 l'École secondaire Saint-Joseph de Hull, un établissement d'enseignement secondaire privé pour filles uniquement. En 2001, l'École prit le nom de Collège Saint-Joseph de Hull, n'accueillant encore aujourd'hui que des filles et dirigé par une corporation religieuse et laïque. ${ }^{79}$

\section{Conclusion}

Notre étude a porté sur les circonstances entourant la création de cette École, sur son évolution de 1908 à 1968, sur ses dirigeants, son personnel enseignant, ses programmes d'études et, bien sûr, sur ses élèves.

Les Sœurs Grises de la Croix d'Ottawa, une congrégation religieuse bien implantée en Outaouais, assurèrent sans interruption la formation d'institutrices d'écoles primaires et secondaires. S'adaptant aux conditions du milieu et aux changements des programmes de formation des maîtres, la congrégation offrit rapidement, malgré les lourdes responsabilités financières que représentait la tenue de cette École et l'éloignement des grands centres québécois, les différents brevets d'enseignement autorisées par les autorités gouvernementales.

Cet enseignement, d'abord dirigé et assumé par un principal appartenant au clergé et par un professeur laïc, fut progressivement donné par les religieuses. Il était offert à une clientèle de plus en plus nombreuse, d'origine géographique et sociale diverse, provenant de milieux ruraux et urbains.

Munies d'un brevet plus ou moins élevé selon leurs talents, leurs ambitions, la situation financière de leur famille et les attentes de leurs milieux, les normaliennes issues de l'École St-Joseph se consacrèrent en majorité à l'enseignement. Pour bon 
nombre, ce sera une carrière et, pour plusieurs d'entre elles, également une vocation.

En définitive, dirigée par une communauté religieuse qui avait rapidement voulu offrir diverses possibilités éducatives aux jeunes filles de la ville et de la région, l'École normale Saint-Joseph de Hull dispensa une formation qui, selon nous, répondait aux exigences du département de l'Instruction publique, aux attentes et aux besoins du milieu ainsi qu'aux aspirations des normaliennes. Cette expérience qui aura duré six décennies nous apparaît, somme toute, une réussite éducative.

\section{Notes}

J'aimerais remercier ici $\mathrm{M}^{\mathrm{me}}$ Gabrielle Marchand-Dauphin, archiviste en chef du centre d'Archives des Sours de la Charité d'Ottawa, pour son obligeance à me fournir des documents d'archives concernant l'École normale Saint-Joseph de Hull. Mes remerciements vont également à Sœur Germaine Julien.

1 Montréal : Arbour et Dupont, 1909.

2 Jeannette Létourneau, Les écoles normales de filles au Québec, coll. " Histoire et documents" (Montréal : Fides, 1981).

3 Thérèse Hamel, Un siècle de formation des maîtres au Québec : 1836-1939 (Montréal : Éditions Hurtubise HMH, 1995). Ajoutons la thèse de doctorat de Roland Piquette intitulée Les programmes de formation des maîtres dans les écoles normales françaises du Québec, 1857-1970 (Montréal : Université de Montréal, 1973).Les ouvrages de Claude Lessard et Maurice Tardif, La profession enseignante au Québec (1945-1990). Histoire, structure, système (Montréal : PUM, 1996) et de M'Hammed Mellouki, Savoir enseignant et idéologie réformiste, 1930-1964 (Québec: IQRC, 1989.

4 On remarquera la périodisation de l'étude de T. Hamel. C'est que, en 1836, fut promulgué l'Acte pour pourvoir à l'établissement d'écoles normales au Bas-Canada. Toutefois adoptée dans un contexte politique difficile et ayant donné fort peu de résultats, cette loi n'est pas considérée comme le point de départ des écoles normales du Québec.

5 Mentionnons cependant que Jeannette Létourneau aborde ponctuellement plusieurs écoles normales, notamment pour rappeler leur contexte de fondation et quelques éléments de leur évolution.

6 Réal Bertrand, L'école normale Laval. Un siècle d'histoire, 1857-1957 (Québec : Société historique de Québec, Cahiers d'histoire n 9, 1957).

7 Une section féminine était toutefois tenue par les religieuses Ursulines à leur Couvent de Québec.

8 Georgette Grand'Maison. Au service du Témiscouata-L'école normale Sainte-Rose de Dégelis (Rimouski : Presses de Saint-Rosaire, 1980).

9 Hamel, Un siècle de formation des maîtres, 185. Louis-Philippe Audet, Histoire de l'enseignement au Québec, t. 2, 1840-1970 (Montréal : Holt, Rinehard et Winston, 1971) : 312; Létourneau, Les écoles normales de filles, 35-36.

10 Létourneau, Les écoles normales de filles, 55.

11 P. Cazes, Règlements refondus du Comité catholique du Conseil de l'Instruction publique de la province de Québec (Québec : 1906, 124; Circulaire No 1. (15 mars 1898) du Bureau central des examinateurs catholiques.

12 Létourneau, Les écoles normales de filles, 55-56, 62-74, 208.

13 Voir notamment Létourneau, Les écoles normales de filles, 55-62 et son Tableau IV.-Écoles normales de jeunes filles par diocèse, p. 215-217. 
14 Depuis 1968, les religieuses ont repris le nom officiel de la communauté, les Sœurs de la Charité d'Ottawa. Nous avons préféré utiliser l'appellation plus connue de Sœurs Grises de la Croix d'Ottawa.

15 Pour un bref historique des Sours Grises de la Croix d'Ottawa, voir Andrée Dufour, " Le Collège Marguerite-d'Youville de Hull, 1945-1964. Un collège classique féminin en milieu ouvrier ", dans Histoire sociale-Social History, vol.47, n 93 (mai 2014), 63-80.

16 Archives de l'École secondaire Saint-Joseph de Hull (AESSJH). Annuaire de l'École normale de Hull 1909-1910, 9.

17 Selon Claudette Laserre, L'éducation des filles au Québec dans la première moitié du XXe siècle. Examen de synthèse pour le doctorat en éducation (Université de Montréal, 1982), 44.

18 Cité par Létourneau, Les écoles normales de filles, 100.

19 Archives de la Maison provinciale Notre-Dame de Hull (AMPNDH) Chroniques $d u$ Couvent Notre-Dame de Grâces ; AESSJH, Annuaire de l'école normale de Hull, 19091910, p. 10.

20 AESSJH, Programme souvenir 1909-1959. Jubilé École normale de Hull, 21-22.

21 Voir Roger Blanchette, L’Outaouais (Québec : Les Presses de l'Université Laval, 2009), 108-109.

22 Létourneau, Les écoles normales de filles, 67-68, 151.

23 Ibid., 151-152.

24 AESSJH, Programme souvenir, 23 ; Létourneau, Les écoles normales de filles, 144, 165.

25 Audet, Histoire de l'enseignement au Québec, 314-317 notamment.

26 ASCO, Annuaire de l'École normale de Hull, 1909-1910, p. 20-22 ; Létourneau, Les écoles normales de filles, 35 et 103.

27 On peut voir les Tableaux de l'emploi du temps, annuaire 1909-1910 (ASCO) dans l'annexe de cet article sur le site de la revue : historicalstudiesineducation.ca.

28 ASCO, M18, SA, SSI, D01.001, D0I.002 et D01.003. Ce règlement peut être vu sur le site de la revue cité plus haut.

29 Pour les matières à l'examen des candidats aux brevets décernés par le Bureau central des examinateurs, voir Hamel, Un siècle de formation, 181, 189-191.

30 AESSJH, Annuaire de l'École normale de Hull, 1909-1910, p. 19.

31 AESSJH, Programme Souvenir..., 10. Entrevue de Sœur Clotilde Maurice du 21 février 1983.

32 Hamel, Un siècle de formation des maîtres, 329.

33 Létourneau, Les écoles normales de filles, 105.

34 AESSJH, Annuaire de l'École normale de Hull, 1923-1924.

35 Létourneau, Les écoles normales de filles au Québec, 105.

36 En 1929, le Comité catholique de l'Instruction publique avait créé le cours primaire supérieur : trois années d'études s'ajoutaient, portant à 11 ans la durée de la scolarité à l'école publique (Audet, Histoire de l'enseignement au Québec, tome 2, 273.

37 C.J. Magnan « Rapport de l'Inspecteur général des Écoles normales catholiques de la province de Québec ", présent dans le Rapport du surintendant de l'Instruction publique 1937-1938, 207, et repris par Létourneau, Les écoles normales de filles au Québec, 106.

38 Létourneau, Les écoles normales de filles au Québec, 107. Ce programme fit vraisemblablement suite au «Rapport de l'Inspecteur général des Écoles normales catholiques de la province de Québec " paru dans le Rapport du surintendant de l'Instruction publique, 1937-1938.

39 AESSJH, Programme Souvenir..., 23.

40 La photographie comprend les neuf étudiantes et le principal, l'abbé Paul Desjardins. On peut lire la devise des étudiantes : « Excelsior ». Dans la première rangée, on aperçoit de gauche à droite Constance Joanette, Clotilde Denis, Monique Groulx, Rachel Lévesque, Huguette Dagenais, et dans la deuxième rangée, Louise Bernier, Denise Schnobb, Marie-Marthe Jacob, Marie-Marthe Bénard. ASCO, P-M18-242. 
41 Létourneau, Les écoles normales de filles au Québec, 169.

42 Audet, Histoire de l'enseignement, 274.

43 AMPNDH, Le Droit, 4 juillet 1956.

44 AMPNDH, Le Droit, juin 1961.

45 Travaux publiés dans le Rapport de la Commission royale d'enquête sur l'enseignement ans la province de Québec, 1965.

46 Voir notamment à ce propos, Thérèse Hamel, «L'enseignement d'hier à aujourd'hui. Les transformations d'un métier «féminin " au Québec dans Identités féminines : mémoire et création (Ville Saint-Laurent : Institut québécois de recherche sur la culture, 1986), 51-70.

47 Avant que les maîtres ne soient formés à l'université, le gouvernement avait imposé des fusions d'institutions d'enseignement, soit des collèges classiques et des écoles normales. On peut penser que ce fut le cas pour les écoles normales de Hull, vu que les trois collèges classiques de la ville et de la région durent fusionner. Voir Andrée Dufour, « Le Collège Marguerite-d'Youville de Hull, 67-80.

48 Entrevue de Sœur Rita Denis, dernière principale de l'École normale, le $1^{\text {er }}$ mars 1983.

49 AESSJH, Annuaire de l'École normale de Hull, 1909-1921, 1922-1933 et 1953 ; Programme Souvenir..., op. cit; entrevue de Sœur Clotilde Maurice, le 21 février 1983 à la Maison provinciale Notre-Dame de Hull.

50 Entrevues de Sœur Clotilde Maurice et de Sœur Rita Denis, 21 février 1983.

51 Arrêté en Conseil 2318/98 du 18 juin 1899.

52 AESSJH, Annuaires ... 1909-1921, 1922-1933, 1953 et entrevue de Sœur Clotilde Maurice, le 21 février 1983.

53 AESSJH, Annuaires ... 1909-1921, 1922-1933, 1953. ASCO, P-M18-071.

54 Dufour, "Le Collège Marguerite-d'Youville », 65-80.

55 AESSJH, Annuaires ... 1953.

56 AESSJH, Programme Souvenir, 37.

57 AESSJH, Annuaire 1909-1910, 5.

58 AESSJH, Annuaire 1929-1930, 4-5. Depuis 1911 cependant, il fallait avoir 17 ans le 30 décembre pour obtenir un brevet d'enseignement (Létourneau, Les écoles normales de filles au Québec, 111).

59 AESSJH, Annuaire 1929-1930, 4-5

60 Le ministère de l'Éducation ne sera établi au Québec qu'en 1964. Entre temps, le Conseil de l'Instruction publique, créé en 1856, avait mis sur pied deux comités confessionnels, un Comité catholique et un Comité protestant, lesquels assumaient la direction générale, le contrôle de l'enseignement, l'organisation et la discipline scolaires. (Audet, Histoire de l'enseignement au Québec, t.2, 339-346.

61 AESSJH, Annuaire 1909-1910, 6.

62 AESSJH, Annuaire 1929-1930, 5.

63 Létourneau, Les écoles normales de filles, 164.

64 ASCO, document P-M18-008.

65 AESSJH, Annuaires 1909-1910, 1929-1930. Entrevue de Sour Clotilde Maurice du 18 février 1983.

66 AESSJH, Annuaire 1953, 13-24.

$67 \mathrm{AESSJH}$, Vingt-cinquième anniversaire de l'École normale St-Joseph et Troisième conventum de l'Amicale, 1934, p. 11.

68 Létourneau, Les écoles normales de filles, 111.

69 Le Collectif Clio, Histoire des femmes au Québec depuis quatre siècles. Coll. "Idéelles " (Montréal : Quinze, 1982), 315, 392.

70 Létourneau, Les écoles normales de filles, 176.

71 Lasserre, L'éducation des filles au Québec, 48. 
72 Répertoire des institutions canadiennes d'enseignement français, 1962-1963 (Québec: Les Éditions L'ACELF, 1963) : 211.

73 AMPNDH, article de La Tribune, 22 juin 1961.

74 Entrevue de Sœur Clotilde Maurice le 8 mars 1983 à la Maison Notre-Dame de Hull.

75 Ces derniers pourcentages ont été calculés par Jeannette Létourneau. Voir Les écoles normales de filles, 212-214.

76 Mémoire des Collèges classiques de jeunes filles à la Commission royale d'enquête sur les problèmes constitutionnel. La Signification et les Besoins de l'enseignement classique pour jeunes filles (Montréal : Fides, 1954), 104.

77 AMPNDH, Deuxième Conventum de l'Amicale de l'École normale de Hull, 1929, p. 3.

78 AESSJH, Vingt-cinquième anniversaire de l'École normale St-Joseph, 11.

79 Site internet du Collège (www.collegestjoseph.ca/college/historique). 